Pensamiento Crítico $N^{\circ} 16$, pp. 51-67

\title{
Free cash flow y el valor de la empresa
}

\author{
Dr. Raimundo Renaun Pacheco Mexzon
}

\section{RESUMEN}

La globalización de la economía y la internacionalización de los mercados de capitales es hoy en día un fenómeno totalmente generalizado en el mundo, por esta razón no hay lugar para aislamiento de los distintos mercados incluyendo el mercado de valores y en el caso del mercado peruano no se escapa a esta realidad. En este nuevo contexto existe un fenómeno muy presente relacionado a la adquisición y fusión de empresas.

La importancia que han tomado estas adquisiciones y fusiones de empresas hace pensar en una técnica de valorización que permita calcular el valor de la empresa para que se pueda realizar esta operación sin problemas y a un precio justo.

De allí que nuestra inquietud es plantear un modelo sencillo para calcular el valor de la empresa partiendo del "Flujo de Caja Libre" cuyo nombre en inglés es "Free Cash Flow".

Palabras claves: Flujo de caja libre, Valor de la empresa.

\section{ABSTRACT}

The globalization of the economy and the internationalization of capital markets today is quite widespread in the world, that is why there is no place for insulation of different markets, including the stock market and in the case of the Peruvian market is no exception to this reality. In this new context exist a phenomenon of acquisition and merger. 


\section{Pensamiento Crítico $\mathbf{N}^{\circ} 16$}

The importance that they have taken these acquisitions and mergers of companies makes us think of a technique of recovery that makes it possible to calculate the value of the company to be able to perform this operation without problems and at a fair price.

Hence, our concern is to raise a simple model to calculate the value of the company based on the "Free Cash Flow".

Keywords: Free Cash Flow, The company's value.

\section{Introducción}

El presente trabajo se desarrolló luego de plantearnos las siguientes preguntas:

1. ¿Las adquisiciones y fusiones de empresas se pueden realizar calculando el valor de la empresa en el mercado?

2. ¿El flujo de caja libre puede ser útil para determinar el valor de la empresa a adquirir o fusionar?

Para responderlas se desarrolló el marco teórico que incluye la conceptualización del valor de la empresa a partir del flujo de caja libre, planteándose un modelo sencillo de valorización.

Luego, se plantea la aplicación del modelo desarrollado en el marco teórico tomando información real de una empresa peruana que cotiza en nuestra Bolsa de Valores de Lima. Esta información que aparece en sus estados financieros auditados, permitió hacer las proyecciones respectivas y llegar a la determinación del valor de la empresa.

Se concluye que el flujo de caja libre proyectado es una buena alternativa para calcular el valor de la empresa a adquirir o fusionar. 


\section{Raimundo Renaun Pacheco Mexzon}

Se recomienda que las empresas peruanas que deseen adquirir o fusionarse con otras empresas utilicen esta metodología para calcular el valor de la empresa.

\section{Marco teórico}

Para calcular el valor de la empresa se presentan una serie de métodos que consideran en términos generales que la empresa tiene una estructura financiera determinada. Es decir el total de activos de la empresa está financiado con pasivos o deudas y patrimonio o capital propio.

Por lo tanto partiendo de la teoría del valor de la empresa en el mercado se puede maximizar este valor teniendo una estructura adecuada de capital.

La representación matemática para calcular el valor de la empresa en el mercado es:

$$
\text { V.E. = VPN + UT.ACU + PLP + PCP }
$$

Donde:

V.E. $\quad=$ Valor de la empresa en el mercado

VPN. = Valor presente neto

UT. ACU = Utilidades acumuladas

PLP = Pasivos de largo plazo

PCP = Pasivos de corto plazo

Pero se conoce que:

VPN + UT. ACU. $=$ PATRIMONIO

$\mathrm{PLP}+\mathrm{PCP} \quad=$ DEUDA 


\section{Pensamiento Crítico $\mathrm{N}^{\circ} 16$}

Reemplazando (2) y (3) en (1) Tenemos:

$$
\mathrm{V} . \mathrm{E}=\text { PATRIMONIO + DEUDA }
$$

Donde:

V.E. $\quad=$ Valor de la empresa en el mercado

PATRIMONIO $=$ VPN + UT. ACU.

DEUDA $\quad=$ DLP + DCP

DLP = Deuda de largo plazo

$\mathrm{DCP}=$ Deuda de corto plazo

Contando con el marco teórico anteriormente descrito se puede plantear el siguiente MODELO:

$$
\text { Máx. V.E. }=\mathrm{k} * \mathrm{VE}(\mathrm{PATRIMONIO})+\mathrm{w} * \mathrm{VE}(\mathrm{DEUDA})
$$

Donde:

El máximo valor de la empresa en el mercado (Max. V. E.) es la variable dependiente y la óptima estructura de capital tiene las variables independientes, PATRIMONIO y DEUDA, que determinan la estructura de capital.

Los coeficientes " $k$ " $y$ " $w$ " son los porcentajes del valor de la empresa que definen el PATRIMONIO y la DEUDA necesarios para determinar la estructura adecuada de capital. Ambos suman "uno" porque representan el $100 \%$.

Matemáticamente tenemos: $\mathrm{k}+\mathrm{w}=1$ de donde $\mathrm{w}=1-\mathrm{k}$

Luego el máximo valor de mercado de la empresa se da con la siguiente estructura de capital: 


\section{Raimundo Renaun Pacheco Mexzon}

$$
\mathrm{k} * \text { VE como PATRIMONIO + }(1-\mathrm{k}) * \text { VE como DEUDA }
$$

Como se puede apreciar con esta estructura de capital se determina el PATRIMONIO necesario para maximizar el valor de la empresa.

Conociendo la estructura de capital se puede determinar el "Flujo de Caja Libre" y proyectarlo considerando que la empresa es un negocio en marcha y que al adquirirla seguirá generando flujos de efectivo en el futuro para toda la vida de la empresa.

En consecuencia al utilizar el "Flujo de Caja Libre" se puede determinar el valor de la empresa en el mercado con el siguiente modelo:

$$
\mathrm{VE}=\mathrm{VAPE}+\mathrm{VAPC}
$$

Donde:

$\mathrm{VE}=$ Valor de la empresa en el mercado.

VAPE $=$ Valor actual del periodo explícito.

VARC $=$ Valor actual del periodo continuo.

El periodo explícito está referido a una proyección del flujo de caja libre mínimo por cinco años. Y el periodo continuo, a partir del sexto año hasta el infinito.

\section{Objetivo}

El objetivo general del presente trabajo es demostrar que se puede calcular el valor de la empresa en el mercado utilizando la técnica del valor presente neto del "Flujo de Caja Libre".

Para lo cual se aplicará un modelo que nos permita calcular la estructura adecuada de capital de una empresa y su valor en el mercado. 


\section{Pensamiento Crítico $\mathrm{N}^{\circ} 16$}

\section{Flujo de caja libre (free cash flow)}

\section{Concepto}

El Flujo de Caja Libre (Free Cash Flow) es el flujo de fondos operativo de la empresa que se determina a partir de la utilidad operativa después de impuesto agregándole la depreciación y restándole la inversión en activos y capital de trabajo.

Algunos le llaman a la utilidad operativa después de impuestos como utilidad antes de intereses después de impuestos.

En consecuencia el Flujo de Caja Libre no incluye gastos financieros ni amortización de deudas.

\section{Un flujo de caja libre}

Si quisiéramos conocer la presentación de un Flujo de Caja Libre veamos el siguiente cuadro:

FLUJO DE CAJA LIBRE

\begin{tabular}{|l|c|c|}
\hline \multicolumn{1}{|c|}{ AÑOS } & $\mathbf{1}$ & $\mathbf{2}$ \\
\hline Ingresos por ventas & 1000 & 1200 \\
\hline Costo de ventas & 600 & 750 \\
\hline Utilidad Bruta & 400 & 450 \\
\hline Gastos operativos & 180 & 200 \\
\hline $\begin{array}{l}\text { Utilidad operativa (Utilidad antes de intereses e } \\
\text { impuestos) }\end{array}$ & $\mathbf{2 2 0}$ & $\mathbf{2 5 0}$ \\
\hline Impuesto a la renta (30\%) & 66 & 75 \\
\hline Utilidad antes de intereses después de impuestos & $\mathbf{1 5 4}$ & $\mathbf{1 7 5}$ \\
\hline (+) Depreciación & 50 & 60 \\
\hline (-) Inversiones en activos fijos & 70 & 30 \\
\hline (-) Incremento del capital de trabajo & 2 & 32 \\
\hline Flujo de caja libre & 132 & 152 \\
\hline
\end{tabular}




\section{Raimundo Renaun Pacheco Mexzon}

\section{Un caso práctico}

En esta parte del trabajo se escogerá una empresa que actualmente cotiza en la Bolsa de Valores de Lima y se le aplicará el modelo planteado en el marco teórico.

De esta manera se determinará su estructura adecuada de capital que permita maximizar su valor en el mercado.

\section{RESUMEN HISTÓRICO DEL BALANCE GENERAL}

En Miles de soles

\begin{tabular}{|c|c|c|c|c|}
\hline & AÑO 4 & AÑO 3 & AÑO 2 & AÑO 1 \\
\hline Activo corriente & 475,171 & 473,222 & 739,462 & 607,125 \\
\hline Caja/bancos & 91,968 & 28,102 & 232,676 & 58,132 \\
\hline $\begin{array}{l}\text { Cuentas por cobrar } \\
\text { comerciales }\end{array}$ & 69,542 & 100,420 & 126,657 & 125,670 \\
\hline Otras cuentas por cobrar & 94,276 & 121,327 & 140,169 & 158,699 \\
\hline Existencias & 201,385 & 223,373 & 239,960 & 264,624 \\
\hline Activo no corriente & $2,037,979$ & $1,917,982$ & $1,618,248$ & $1,834,445$ \\
\hline Activo fijo neto & 802,900 & 801,759 & 861,694 & 902,734 \\
\hline Otras inversiones & 864,976 & 878,378 & 617,212 & 781,043 \\
\hline Intangibles netos & 370,103 & 237,845 & 139,342 & 150,668 \\
\hline Total activos & $2,495,150$ & 2,391204 & $2,357,716$ & $2,441,570$ \\
\hline Pasivo corriente & 301,160 & 303,071 & 422,896 & 399,989 \\
\hline Préstamos bancarios & 17,273 & 83,704 & 25,076 & 107,454 \\
\hline $\begin{array}{l}\text { Cuentas por pagar } \\
\text { comerciales }\end{array}$ & 23,445 & 28,046 & 29,166 & 41,982 \\
\hline Otras cuentas por pagar & 156,754 & 120,210 & 129,088 & 155,383 \\
\hline $\begin{array}{l}\text { Parte corriente deuda } \\
\text { largo plazo }\end{array}$ & 103,688 & 71,111 & 239,566 & 95,165 \\
\hline
\end{tabular}




\section{Pensamiento Crítico $\mathrm{N}^{\circ} 16$}

\begin{tabular}{|l|c|c|c|c|}
\hline Pasivo no corriente & $\mathbf{5 3 4 , 8 9 6}$ & $\mathbf{6 0 2 , 8 8 2}$ & $\mathbf{2 2 9 , 3 5 4}$ & $\mathbf{3 2 2 , 6 2 7}$ \\
\hline Deuda a largo plazo & 512,405 & 576,641 & 199,546 & 315,772 \\
\hline Otros pasivos & 22,491 & 26,246 & 29,808 & 6,855 \\
\hline Total pasivos & $\mathbf{8 3 6 , 0 5 6}$ & $\mathbf{9 0 5 , 9 5 8}$ & $\mathbf{6 5 2 , 2 5 0}$ & $\mathbf{7 2 2 , 6 1 6}$ \\
\hline Patrimonio & $\mathbf{1 , 6 5 9 , 0 9 4}$ & $\mathbf{1 , 4 8 5 , 2 4 6}$ & $\mathbf{1 , 7 0 5 , 4 6 0}$ & $\mathbf{1 , 7 1 8 , 9 5 4}$ \\
\hline Capital social & $1,003,482$ & $1,003,482$ & $1,003,482$ & 967,227 \\
\hline Acciones de inversión & 491,174 & 491,174 & 491,174 & 473,429 \\
\hline Reservas y otros & 140,316 & $(37,005)$ & 185,286 & 203,542 \\
\hline Resultados acumulados & 24,122 & 27,595 & 25,518 & 74,756 \\
\hline $\begin{array}{l}\text { Total pasivos y } \\
\text { patrimonio }\end{array}$ & $\mathbf{2 , 4 9 5 , 1 5 0}$ & $\mathbf{2 , 3 9 1 , 2 0 4}$ & $\mathbf{2 , 3 5 7 , 7 1 0}$ & $\mathbf{2 , 4 4 1 , 5 7 0}$ \\
\hline
\end{tabular}

Con estos datos históricos podemos presentar el comportamiento que han tenido los coeficientes " $\mathrm{k}$ " y "w" en el periodo del AÑO 1 al AÑO 4 tal conforme se indican a continuación:

\begin{tabular}{|c|c|c|c|c|}
\hline COEFICIENTE & AÑO 4 & AÑO 3 & AÑO 2 & AÑO 1 \\
\hline "k" & 0.66 & 0.62 & 0.72 & 0.70 \\
\hline$" w "$ & 0.34 & 0.38 & 0.28 & 0.30 \\
\hline "k" + "w" & 1.00 & 1.00 & 1.00 & 1.00 \\
\hline
\end{tabular}

Del comportamiento de los coeficientes " $k$ " y " $w$ " podemos inferir que de acuerdo a los resultados obtenidos por la empresa la estructura de capital ha sufrido modificaciones muy importantes pasando de una relación de $70 \%$ de capital y $30 \%$ de deuda en el AÑO 1, a una estructura adecuada de capital de $66 \%$ de patrimonio y 34 $\%$ de deuda en el año 4.

A continuación se presenta el resumen del estado de ganancias y pérdidas para el mismo periodo. 
Raimundo Renaun Pacheco Mexzon

\section{RESUMEN HISTÓRICO DEL ESTADO DE GANANCIAS Y PÉRDIDAS}

En miles de soles

\begin{tabular}{|l|c|c|c|c|}
\hline \multicolumn{1}{|c|}{ RUBROS } & AÑO 4 & AÑO 3 & AÑO 2 & AÑO 1 \\
\hline Ventas netas & 865,787 & 870,716 & 892,863 & 982,512 \\
\hline Otros ingresos & 38,002 & 27,381 & 37,086 & 45,734 \\
\hline TOTAL INGRESOS & $\mathbf{9 0 3 , 7 8 9}$ & $\mathbf{8 9 8 , 0 9 7}$ & $\mathbf{9 2 9 , 9 4 9}$ & $\mathbf{1 , 0 2 8 , 2 4 6}$ \\
\hline Costo de ventas & 332,528 & 350,592 & 374,410 & 401,080 \\
\hline Utilidad bruta & $\mathbf{5 7 1 , 2 6 1}$ & $\mathbf{5 4 7 , 5 0 5}$ & $\mathbf{5 5 5 , 5 3 0}$ & $\mathbf{6 2 7 , 1 6 6}$ \\
\hline Gastos operativos & 312,570 & 325,213 & 365,605 & 381,089 \\
\hline Utilidad operativa & $\mathbf{2 5 8 , 6 9 1}$ & $\mathbf{2 2 2 , 2 9 2}$ & $\mathbf{1 8 9 , 9 3 4}$ & $\mathbf{2 4 6 , 0 7 7}$ \\
\hline Otros ingresos y egresos & 17,525 & 36,548 & 59,852 & 59,787 \\
\hline $\begin{array}{l}\text { Utilidad entes de } \\
\text { participación }\end{array}$ & $\mathbf{1 9 1 , 1 6 6}$ & $\mathbf{1 8 5 , 7 4 4}$ & $\mathbf{2 4 9 , 7 8 6}$ & $\mathbf{1 8 6 , 2 9 0}$ \\
\hline Participaciones & 22,581 & 20,468 & 21,251 & 19,091 \\
\hline $\begin{array}{l}\text { Utilidad antes de } \\
\text { impuestos }\end{array}$ & $\mathbf{1 6 8 , 5 8 5}$ & $\mathbf{1 6 5 , 2 7 6}$ & $\mathbf{2 2 8 , 5 3 5}$ & $\mathbf{1 6 7 , 1 9 9}$ \\
\hline Impuesto a la renta & 52,010 & 54,987 & 44,794 & 51,233 \\
\hline Utilidad neta & $\mathbf{1 1 6 , 5 7 5}$ & $\mathbf{1 1 0 , 2 8 9}$ & $\mathbf{1 8 3 , 7 4 1}$ & $\mathbf{1 1 5 , 9 6 6}$ \\
\hline
\end{tabular}

De acuerdo a los datos históricos se aprecia que los ingresos por ventas han tenido un comportamiento cíclico llegando en el año 4 a 865 millones 787 mil soles. La utilidad operativa llegó a 258 millones 691 mil soles y la utilidad neta a 116 millones 575 mil soles.

Tomando como base la data histórica, se calculará el valor de la empresa, en dólares, considerando un escenario futuro de cinco años como periodo explícito y las siguientes políticas: 


\section{Pensamiento Crítico $\mathrm{N}^{\circ} 16$}

Mantener el crecimiento de la utilidad antes de intereses y de impuestos, para lo cual, deben crecer las ventas de tal manera de llegar a su mejor nivel alcanzado en un año histórico (Aproximadamente 273 millones de dólares). Entonces el crecimiento de las ventas tendrá el siguiente comportamiento:

PROYECCIONES DE LAS VENTAS (EN MILES DE US\$)

\begin{tabular}{|c|c|c|c|c|c|c|}
\hline AÑOS & $\mathbf{0}$ & $\mathbf{1}$ & $\mathbf{2}$ & $\mathbf{3}$ & $\mathbf{4}$ & $\mathbf{5}$ \\
\hline Ventas & 240,441 & 252,463 & 257,512 & 262,662 & 267,916 & 273,274 \\
\hline Crecimiento & & $5 \%$ & $2 \%$ & $2 \%$ & $2 \%$ & $2 \%$ \\
\hline
\end{tabular}

El costo de ventas que ha tenido un comportamiento histórico entre el $41 \%$ y el $38 \%$ de las ventas, debe llegar como máximo a un $40 \%$. Por lo tanto el costo de ventas tendrá el siguiente comportamiento:

PROYECCIONES DEL COSTO DE VENTAS EN MILES DE US\$

\begin{tabular}{|c|c|c|c|c|c|}
\hline AÑOS & $\mathbf{1}$ & $\mathbf{2}$ & $\mathbf{3}$ & $\mathbf{4}$ & $\mathbf{5}$ \\
\hline Costo de Ventas & 100,985 & 103,005 & 105,065 & 107,166 & 109,310 \\
\hline $\begin{array}{c}\text { Porcentaje de } \\
\text { Ventas }\end{array}$ & $40 \%$ & $40 \%$ & $40 \%$ & $40 \%$ & $40 \%$ \\
\hline
\end{tabular}

Los gastos operativos que han tenido un comportamiento histórico entre $37 \%$ y $40 \%$ del costo de ventas, deben llegar como máximo a un $38 \%$, por lo tanto tendrán el siguiente comportamiento:

PROYECCIONES DE LOS GASTOS OPERATIVOS EN MILES DE US\$

\begin{tabular}{|c|c|c|c|c|c|}
\hline AÑOS & $\mathbf{1}$ & $\mathbf{2}$ & $\mathbf{3}$ & $\mathbf{4}$ & $\mathbf{5}$ \\
\hline $\begin{array}{c}\text { Gastos } \\
\text { Operativos }\end{array}$ & 38,374 & 39,142 & 39,925 & 40,723 & 41,538 \\
\hline $\begin{array}{c}\text { Porcentaje del } \\
\text { Costo de Ventas }\end{array}$ & $38 \%$ & $38 \%$ & $38 \%$ & $38 \%$ & $38 \%$ \\
\hline
\end{tabular}




\section{Raimundo Renaun Pacheco Mexzon}

El capital de trabajo que históricamente llegó a 43 millones 340 mil dólares, debe de crecer acorde con las ventas, es decir $5 \%$ el primer año y $2 \%$ del segundo al quinto año. Por lo tanto el crecimiento del capital de trabajo será:

\section{CRECIMIENTO DEL CAPITAL DE TRABAJO EN MILES DE US\$}

\begin{tabular}{|c|c|c|c|c|c|}
\hline AÑOS & $\mathbf{1}$ & $\mathbf{2}$ & $\mathbf{3}$ & $\mathbf{4}$ & $\mathbf{5}$ \\
\hline $\begin{array}{c}\text { Incremento } \\
\text { del Capital de } \\
\text { Trabajo }\end{array}$ & 2,167 & 2,210 & 2,254 & 2,299 & 2,345 \\
\hline
\end{tabular}

Históricamente la empresa invirtió en modernización de sus instalaciones 30 millones de soles, equivalente a 8 millones 333 mil dólares. Este tipo de inversión que es necesaria para mantener sin problemas el proceso productivo se estima que la empresa la ejecutará en el tercer año del periodo explícito, incrementando de esta manera el activo fijo. La depreciación de este tipo de activos se realizará en 5 años a razón de 20 $\%$ anual.

Con esta información el flujo de caja libre, generado por la empresa en los próximos cinco años, sería:

FLUJO DE CAJA LIBRE PROYECTADO PARA EL PERIODO EXPLÍCITO EN MILES DE US\$

\begin{tabular}{|c|c|c|c|c|c|}
\hline AÑOS & $\mathbf{1}$ & $\mathbf{2}$ & $\mathbf{3}$ & $\mathbf{4}$ & $\mathbf{5}$ \\
\hline Ventas & 252,463 & 257,512 & 262,662 & 267,916 & 273,274 \\
\hline Costo de Ventas & 100,985 & 103,005 & 105,065 & 107,166 & 109,310 \\
\hline Gastos Operativos & 38,374 & 39,142 & 39,925 & 40,723 & 41,538 \\
\hline $\begin{array}{c}\text { Utilidad Antes } \\
\text { de Intereses e } \\
\text { Impuestos }\end{array}$ & 113,103 & 115,365 & 117,673 & 120,026 & 122,427 \\
\hline $\begin{array}{c}\text { Utilidad Antes de } \\
\text { Intereses después } \\
\text { de Impuestos (Tasa } \\
\text { 30\%) }\end{array}$ & 79,172 & 80,756 & 82,371 & 84,018 & 85,699 \\
\hline
\end{tabular}


Pensamiento Crítico $\mathrm{N}^{\circ} 16$

\begin{tabular}{|c|c|c|c|c|c|}
\hline (+) Depreciación & 9,888 & 9,888 & 9,888 & 11,554 & 11,556 \\
\hline $\begin{array}{c}\text { (-) Desembolso } \\
\text { de capital por } \\
\text { Inversiones }\end{array}$ & 9,888 & 9,888 & 18,221 & 11,554 & 11,556 \\
\hline $\begin{array}{c}\text { (-) Incremento del } \\
\text { Capital de Trabajo }\end{array}$ & 2,167 & 2,2102 & 2,254 & 2,299 & 2,345 \\
\hline Flujo de Caja Libre & $\mathbf{7 7 , 0 0 6}$ & $\mathbf{7 8 , 5 4 6}$ & $\mathbf{7 1 , 7 8 3}$ & $\mathbf{8 1 , 7 1 9}$ & $\mathbf{8 3 , 3 5 3}$ \\
\hline
\end{tabular}

Para traer a valor presente el flujo de caja libre debemos de calcular previamente el costo promedio ponderado de capital (WACC: por sus siglas en inglés) utilizando la siguiente fórmula:

$$
\mathrm{WACC}=\mathrm{P} /(\mathrm{P}+\mathrm{D}) *(\mathrm{~T} \operatorname{lr}+\mathrm{B} * \mathrm{PRM})+\mathrm{D} /(\mathrm{P}+\mathrm{D}) * \mathrm{kd}(1-\mathrm{T})
$$

Donde:

$\mathrm{P} \quad=$ Patrimonio

D = Deuda

TIr = Tasa libre de riesgo

B = Coeficiente beta

$\mathrm{PRM}=$ Prima del mercado $=($ Rendimiento del mercado $-\mathrm{Tl} r)$

$\mathrm{Kd}=$ Costo de la deuda

$\mathrm{T} \quad=$ Tasa de impuesto a la renta 


\section{Raimundo Renaun Pacheco Mexzon}

\section{Cálculo del costo del capital propio}

Para calcular el costo del capital propio se suele utilizar las condiciones presentadas en el mercado considerándose los riesgos respectivos. Es a partir del modelo CAPM (Capital Assets Pricing Model) que se puede calcular una tasa de rendimiento esperada para el accionista (Tresp), que estaría definiendo su costo de oportunidad. El modelo que se resume en la siguiente fórmula nos permitió calcular el costo del capital propio.

$$
\text { Tresp }=\mathrm{TLr}+\mathrm{B}(\mathrm{RPM}-\mathrm{TLr})
$$

TLr, es la tasa libre de riesgo y se asumió que su valor en el mercado es de 5.53 $\%$ tomando en consideración el rendimiento que pagan los bonos en el mercado americano.

(RPM - TLr), es la prima del mercado (PRM) y para el caso de empresas peruanas está relacionada con la diferencia entre lo que pagan los Bonos Brady y la tasa del Bono Americano. En este caso se asumió que es de $5.7 \%$. Además hay que tener en cuenta que en el Perú para cualquier actividad se podría utilizar este valor ya que en el mercado americano se considera como prima de riesgo y ha sido calculado como el promedio geométrico del índice S \& P 500.

"B" conocido como beta, es el factor que determina las condiciones de riesgo con las probabilidades de rendimientos esperados en el mercado a través de la relación de la covarianza y la varianza. En el mercado americano se estima para las empresas del sector de extracción de recursos naturales y sin apalancamiento un beta promedio de 1.7, sin embargo para empresas líderes el beta oscila entre menor que uno y 1.2 dependiendo del riesgo. Como nuestra empresa es una empresa líder en el Perú, el beta considerado es de 1.06 que resulta de ajustar 1.7 a un nivel de riesgo manejable del $62.35 \%$. $(1.7 * 0.6235=1.06)$.

En consecuencia la tasa de rendimiento esperada como costo del capital propio para nuestra empresa se estimó en: 


\section{Pensamiento Crítico $\mathrm{N}^{\circ}$ I6}

$$
\begin{gathered}
\text { Tresp. }=\mathrm{TLr}+\mathrm{B}(\mathrm{RPM}-\mathrm{TLr}) \\
\text { Tresp. }=5.53+1.06 *(11.23-5.53) \\
\text { Tresp. }=11.57 \%
\end{gathered}
$$

\section{Cálculo del costo de la deuda}

Para calcular el costo de la deuda se han tomado los datos históricos de los estados financieros. Se determinó una tasa en soles de $9.45 \%$ anual. Esta tasa ajustada a tasa equivalente en dólares, nos dio una tasa de 3. $15 \%$. En consecuencia la tasa para la deuda se estimó en $3.15 \%$ anual. Ver cuadro siguiente:

\begin{tabular}{|l|c|}
\hline \multicolumn{1}{|c|}{ RUBROS } & MILES DE SOLES \\
\hline Gastos financieros & $\mathbf{6 9 , 1 9 1}$ \\
\hline Total deuda & $\mathbf{7 3 1 , 4 5 6}$ \\
\hline 1. Préstamos bancarios & 83,704 \\
\hline 2. Vencimiento corriente deuda largo plazo & 71,111 \\
\hline 3. Deuda a largo plazo & 576,641 \\
\hline Gastos financieros ENTRE Deuda & $\mathbf{9 . 4 5} \%$ \\
\hline Costo promedio deuda en US\$ & $6.10 \%$ \\
\hline Tasa equivalente en US\$ & $\mathbf{( 1 + 9 . 4 5} \%) /(\mathbf{1 + 6 . 1 0} \%)=\mathbf{3 . 1 5} \%$ \\
\hline
\end{tabular}

De acuerdo a la información obtenida los valores para calcular el WACC son:
$P /(P+D)=66 \%$
$\mathrm{D} /(\mathrm{P}+\mathrm{D})=34 \%$
$\operatorname{Tlr} \quad=5.53 \%$
$\mathrm{B}=1.06$ 


\section{Raimundo Renaun Pacheco Mexzon}

$\begin{array}{ll}\text { PRM } & =5.70 \% \\ \mathrm{Kd} & =3.15 \% \\ \mathrm{~T} & =30 \%\end{array}$

Al reemplazar los datos en la fórmula se obtiene un WACC de $8.39 \%$. De esta manera el valor actual del flujo de caja libre para el periodo explícito se calcula de la siguiente manera:

\section{VALOR ACTUAL DEL FLUJO DE CAJA LIBRE PARA EL PERIODO EXPLÍCITO} (VAPE)

\begin{tabular}{|c|c|c|c|}
\hline AÑOS & FLUJO & $\begin{array}{c}\text { FACTOR } \\
\mathbf{8 . 3 9 \%}\end{array}$ & V A \\
\hline 1 & 77,006 & 0.92262 & 71,047 \\
\hline 2 & 78,546 & 0.85122 & 66,860 \\
\hline 3 & 71,783 & 0.78535 & 56,375 \\
\hline 4 & 81,719 & 0.72458 & 59,212 \\
\hline 5 & 83,353 & 0.66851 & 55,723 \\
\hline VAPE & & & $\mathbf{3 0 9 , 2 1 7}$ \\
\hline
\end{tabular}

Como la empresa es un negocio en marcha se estima que a partir del sexto año habrá un flujo continuo con un crecimiento de las ventas en $1 \%$ por lo tanto para el año 6 el flujo de caja libre será de: $83,353 * 1.01=84,187$. Así mismo el retorno sobre la nueva inversión será de $1 \%$ en forma continua.

Con esta información se calcula el valor continuo que será igual a:

$$
\begin{aligned}
& \mathrm{VC}=\text { FC Libre año } 6 /(\text { WACC }- \text { Retorno nueva inversión) } \\
& \mathrm{VC}=84,187 /(8.39 \%-1 \%) \\
& \mathrm{VC}=1,139 \text { millones } 628 \text { mil dólares }
\end{aligned}
$$




\section{Pensamiento Crítico $\mathrm{N}^{\circ} 16$}

Como el costo de capital promedio ponderado (WACC) es $8.39 \%$ el Valor presente o valor actual de este flujo del periodo continuo sería:

$$
\mathrm{VAPC}=1,139^{\prime} 628 /(1+0.0839) \wedge 6
$$

$$
\mathrm{VAPC}=702,902
$$

Luego el valor de la empresa será:

$\mathrm{VE}=\mathrm{VAPE}+\mathrm{VAPC}$

$\mathrm{VE}=309,217+702,902$

$$
\mathrm{VE}=1,012 \text { millones } 118 \text { mil dólares }
$$

\section{Conclusiones}

1. Utilizando el "Flujo de Caja Libre" y determinando una adecuada estructura de capital se maximiza el valor de la empresa como se demuestra en el caso práctico.

2. Esta técnica nos permite maximizar el valor de la empresa en el mercado considerando que es un negocio en marcha y al venderla o fusionarse con otra empresa se consiguen mejores resultados para los accionistas.

\section{Recomendaciones}

1. Las empresas peruanas que se vendan o se fusionen con otras empresas deben considerar que son un negocio en marcha por lo tanto deben calcular el precio maximizando su valor utilizando la técnica del valor presente de los flujos de caja libre tanto del periodo explícito como del periodo continuo. 


\section{Raimundo Renaun Pacheco Mexzon}

2. Utilizar para maximizar el valor de la empresa el modelo planteado para determinar la estructura óptima de capital.

\section{Bibliografía}

1.ALIAGA L. Rafael y SOUSA DE BARBIERI Lorenzo "Banca de inversión" Edit. Publicaciones Universidad de Piura 1986

2.BELLIDO S. Pedro "Administración Financiera" Edit. Técnico Científica 1989 Capítulo 17: Pags 245 - 272, Capítulo 18: Pags 277 - 297

3.BOLTEN Esteven "Manual de administración financiera". Edit. Ciencia y técnica. Limusa 1987. Capítulo 8: Pags. 351 - 378, Capítulo 10: Pags. 423 - 457

4.DIÉZ DE CASTRO Luís y MASCARREÑAS Juan "Ingeniería financiera: la gestión en los mercados financieros internacionales". Edit. McGRAW-HILL. 1998. Pags 91-92

6.FERNÁNDEZ Pablo "Valoración de empresas" Edit. Gestión 2000 S.A. 1999 Capítulos $1,14,15,16,19$ y 20

7.PACHECO Mexzon Raimundo Renaun ADRs y el Valor de La Empresa" Tesis para optar el Grado Académico de Magister en Economía mención Finanzas Pags 11-13 y $47-50$

8.VAN HORNE James "Administración Financiera" Edit. Contabilidad Moderna Buenos Aires 1973 Capítulo VII: Pags. 260 - 286, Capítulo VIII: Pags. 300 - 313

9.http://www.pla.net.py/cnv/orientando, Glosario

10.http://www.superval.gob.sv/html, Glosario

11.http://www.corfinsura.com/español, Glosario 